\section{Optical Sectioning in Fluorescence Microscopy by Confocal and 2-Photon Molecular Excitation}

\section{Techniques}

M.B. Cannell \& C.Soeller

St. George's Hospital Medical School, Cranmer Terrace, London SW17 ORE

\section{Confocal Microscopy}

Fluorescence microscopy has proved to be an invaluable tool for biomedical science since it is possible to visualise small quantities of labeled materials (such as intracellular ions and proteins) in both fixed and living cells. However, the conventional wide field fluorescence microscope suffers from the disadvantage that objects outside the focal plane also fluoresce (in response to the excitation light) and this leads to a marked loss of contrast for objects in the focal plane. This is especially a problem when the fluorescent probe is distributed throughout the thickness of the cell and the cell is thicker than about $1 \mu \mathrm{m}$. The confocal microscope overcomes this problem by illuminating the preparation with a point source of excitation light and limiting the collection of light with a pinhole that is confocal with the illumination source. This converts the microscope from an imaging system to a point detector and images are produced by scanning the illuminating and detecting point over the specimen to build an image (in much the same way that a television set produces an image). The basic idea behind the confocal system is shown in Fig. 1, and it should be noted that light from points outside the focal plane is defocused at the pinhole and so does not pass through the pinhole efficiently. This leads to the rejection of out-of-focus fluorescence and an improvement in image sharpness (for further details see Pawley, 1995). For fixed preparations this advantage may be limited since it is possible (at least in principle) to use deconvolution techniques to mathematically correct the in-plane image for out-of-focus fluorescence. (With this method, a large number of images at different depths within the specimen are taken and the contribution of out-of-focus fluorescence is calculated and subtracted from data at the image plane.) However, such 'deconvolution' techniques are of limited use when images are needed in near real time or when the specimen itself is changing with time.

The discovery of calcium sparks (Cheng et al., 1993; Cannell et al., $1994,1995)$ was the direct result of the application of the confocal microscope to cardiac calcium metabolism and is a good example of what can be

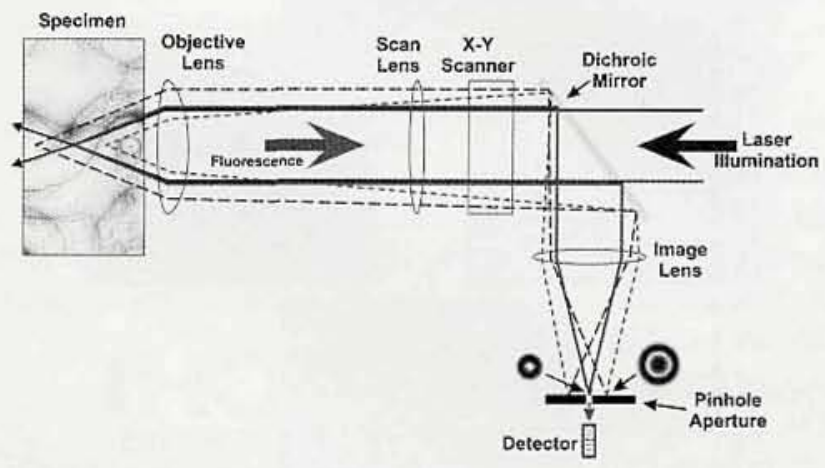

Fig. 1: Schematic drawing of the optical path in a confocal microscope. The laser illumination light (blue) is coupled into the optical system through a dichroic mirror (here a short wave pass mirror appropriate for one-photon excitation). The illuminating beam is scanned across the specimen by an $x-y$ scanner and focused into the specimen by an objective lens. The scan lens ensures that the rear aperture is filled while correcting tube length. Emitted fluorescence (red) is collected by the same objective lens and passes through the $x-y$ scanner ("descanned"). After most of the reflected illumination light has been separated by the dichroic mirror, the fluorescence is imaged onto the pinhole aperture by an image lens. Note how depth discrimination is achieved by the small pinhole aperture which rejects most of the light from locations above and below the plane of focus (dashed green rays). achieved with this methodology. Calcium sparks are microscopic calcium release events inside living muscle cells and their properties are giving new insight into how excitation leads to contraction (Cannell et al., 1995; López-López et al., 1995; Gómez et al., 1997). Although the wide field microscope had been applied to calcium imaging since about 1985, calcium sparks had not been observed previously. This is probably because the presence of fluorescence from outside the focal plane results in a marked loss of in-plane contrast for wide field microscopy. (Note also that fluo-3 was used as the calcium indicator in these experiments as it has low fluorescence in the absence of calcium which also improves image contrast.) The calcium spark illustrates the high sensitivity of current confocal optical methods - the calcium spark finally occupies about $10 \mathrm{ff}$ $\left(10^{-14} \mathrm{I}\right)$ and represents calcium binding to only $\sim 10^{4}$ indicator molecules. Until recently, the laser scanning confocal microscope has been the only instrument that could measure fluorescence with a spatial resolution of about $0.4 \times 0.4 \times 0.8$ $\mathrm{mm}(\mathrm{x}, \mathrm{y}$ and $\mathrm{z}$ respectively) on the millisecond time scale.

\section{2-Photon Molecular Excitation Microscopy.}

Since a major problem in fluorescence imaging resides in the existence of fluorescence from outside the image plane, a solution to this problem would be to prevent fluorescence excitation outside the focal plane. This is exactly what has been achieved with 2- (or multi-photon) photon molecular excitation microscopy which was first demonstrated in 1990 (Denk, et al., 1990). As illustrated in Fig. $2 A$, the excited state is normally achieved by the absorption of a short wavelength photon. After radiationless transitions down the vibrational ladder, fluorescence emission occurs with a transition towards the ground state. The difference in energy between the absorbed photon and the emitted photon leads to Stokes' Law of Fluorescence which states that the wavelength of emitted light is longer than the exciting light. However, it is also possible to achieve the excited state by the near simultaneous absorption of two longer wavelength photons, as shown in Fig. $2 \mathrm{~B}$. The probability of absorbing 2-photons will depend on the square of the

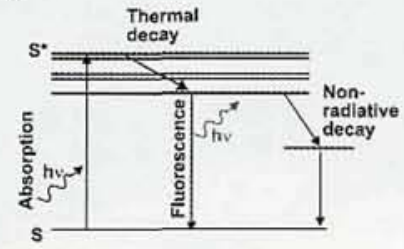

C

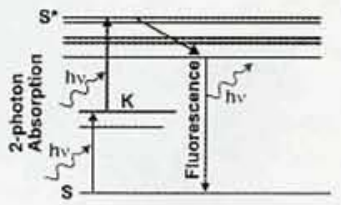

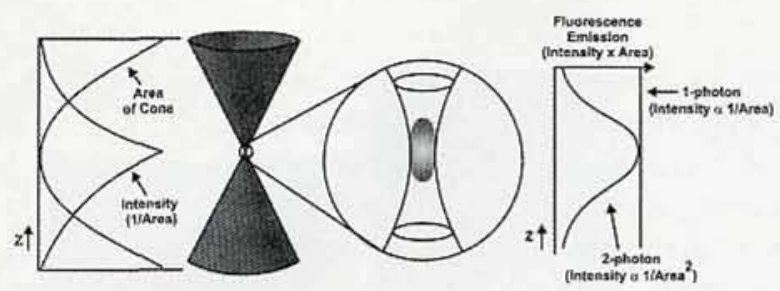

Fig. 2: Principles of one-and two-photon excitation microscopy. Panel A shows a simplified Jablonski diagram of the processes involved in one-photon excitation of a generic fluorochrome molecule. Absorption of an excitation photon raises the molecule from the ground state (S) (which is really a quasi-continuum of vibrational sublevels) to an excited state $\left(\mathrm{S}^{*}\right)$. From there it quickly relaxes to the lowest vibrational sublevel and subsequently decays to the ground state with the emission of a photon of fluorescence. Although most decays lead to the emission of a (red-shifted) photon there is also the possibility of non-radiative decay. The mechanisms of two-photon excitation are similar (panel B). Here, absorption of an exciting (near-infrared) photon raises the molecule to a short-lived "virtual" state from which a second incident photon can raise it to the excited state. The subsequent decay mechanisms are similar to those in one-photon excitation. Photo-bleaching processes, which remove molecules from the pool of fluorochrome molecules, have been omitted for clarity. Panel C illustrates how the different dependence of fluorescence emission on (two photon) excitation intensity results in three-dimensionally localised excitation. In one-photon excitation, the linear dependence of emission on excitation intensity results in any plane having the same contribution to total fluorescence (since excitation intensity $x$ area is the same everywhere). For two-photon excitation (with a square dependence on excitation intensity), the total fluorescence from a given plane decays quickly with increasing distance from the focal plane. 
illumination intensity and for normal light sources this is very low. However, by 'mode-locking' a laser light source it is possible to produce very short, intense light pulses. The short duration of the pulses means that while the average laser power is quite low $(\sim 1 \mathrm{~W})$, the peak pulse powers are very high. For our titanium sapphire (Ti:S) laser based system, peak powers are $>10 \mathrm{~kW}$ in pulses that last $\sim 5 \times 10^{-14} \mathrm{~s}$ with a pulse repetition rate of $\sim 80 \mathrm{MHz}$. The high brightness of the pulses results in a high probability that 2-photons will be able to interact with the fluorochrome and produce the excited state while the lower average power prevents specimen destruction.

By feeding such a light source into an objective lens, the intensity of the light in the illumination cone increases as the focal point is approached (see Fig. 2C). At the focal point, the intensity of the illumination is at a maximum and there the probability of a 2-photon transition taking place is highest. Outside the focal point, the probability of excitation falls with the fourth power of distance (from the probability of excitation being proportional to the square of intensity which is, in turn, proportional to the square of distance) at a rate that depends on the numerical aperture of the objective lens; being highest for high numerical aperture lenses. Thus while conventional illumination results in excitation throughout the whole thickness of the preparation (with every plane producing the same total fluorescence), the squared intensity dependence of 2-photon excitation results in illumination being restricted to the focal plane (Fig. 2C). As shown in Fig. 3 , 2-photon illumination also restricts flurochrome destruction (bleaching) to the focal plane which makes obtaining a large number of optical sections for 3D reconstruction easier (see below).

The lower energy of the photons needed for 2-photon excitation implies a longer excitation wavelength for the fluorochrome; typically the 2-photon excitation maximum will be a little less than twice the wavelength of the one-photon excitation maximum. In other words, it is possible to excite fluorochromes, which are normally excited by near UV or visible light (about $340-600 \mathrm{~nm}$ ), with a tunable infrared Ti:S laser operating in the range $680-1000 \mathrm{~nm}$. It should also be noted that the use of infra-red light for excitation implies that scattering may be reduced in thick specimens. Since the resolution of the microscope is determined only by the properties of the excitation light cone (rather than the optical behaviour of both the illumination and detection systems) there is no need for any pinhole aperture. The removal of the pinhole (and any associated optics) results in a significant improvement in detection efficiency. This gain can be further increased by avoiding the mirror scanning system by feeding light from the rear aperture of the objective directly to the detector with a suitable dichroic mirror. In this way, scattered photons can also be collected and these photons supply useful signal for 2-photon microscopy but only degrade image contrast in confocal microscopy when they arise from the fluorescence which occurs outside the focal point. Despite these advantages, it should be noted that the incident light is very intense and can lead to specimen damage. Where time resolution is not an issue, the damage threshold can be avoided by reducing the excitation light intensity and integrating the output signal for a longer period. While such an approach can be easily applied to fixed specimens, in living specimens that we have used (cardiac myocytes, mouse eggs and skeletal muscle) we have not yet achieved a higher signal to noise ratio (or time resolution) than can be achieved with one photon excitation in conventional confocal methods.

There are additional problems in developing and using a 2-photon excitation
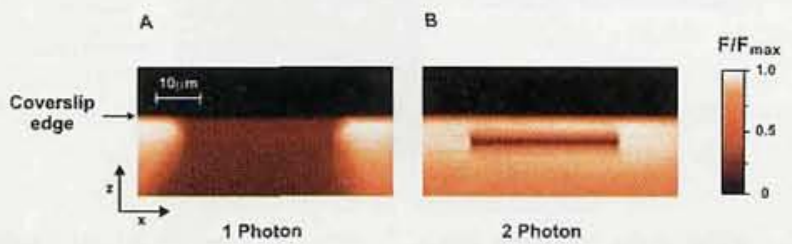

Fig. 3: Photo-bleaching in one-versus two-photon excitation microscopy. Panel A shows the photo-bleaching pattern in a thin gel layer stained with immobilised fluorescein. After repeatedly imaging a plane $\sim 5 \mu \mathrm{m}$ below the coverslip edge, strong bleaching above and below the plane of focus is observed (since the total dosage of excitation light in the double cone of excitation light is independent of distance from the focus). In panel $B$, the photobleaching pattern in an identical sample after repeated imaging in two-photon mode is shown. Here, photo-bleaching is restricted to the immediate vicinity of the focal plane because of the strong axial dependence of the probability of two-photon excitation.

microscope: 1) Accurate alignment of the laser to the microscope is more difficult since the excitation light is invisible (infrared), 2) The optical path of most scanning microscopes is arranged so that long wave pass dichroic mirrors can be used to split the emission light from the excitation light. Switching to a 2-photon source implies that short wave pass mirrors must be substituted and these mirrors have only limited pass bands, 3) The pulse is distorted by the optical elements in the optical train. This problem arises from the speed of light in materials being wave-length dependent, so the relative phase of the wavelength components making up the pulse is changed, resulting in a longer (and lower peak amplitude) pulse. Fortunately, it is possible to use a prism compensator system to pre-distort the pulse in the opposite way to that produced by the lenses in the microscope. Such pre-distortion is then removed by the microscope elements so the pulse shape is restored at the focal point of the objective (Soeller and Cannell, 1996). Such 'pre-chirping' requires an extension of the optical system outside the microscope which increases the overall complexity of the optical design, 4) The mode-locked laser is complicated and requires regular maintenance, 5) Mode-locked laser sources are very expensive and generally require an additional powerful laser as an energy source (in our system we use a $20 \mathrm{~W}$ argon-ion laser as a 'pump' for the Ti:S laser).

Despite the above problems associated with 2-photon microscopy, the restriction of photo-damage to the focal plane is an attractive benefit. This also means that it is also possible to photo-release active molecules within cells from their 'caged' precursors. In this technique, the active molecule is modified by the addition of a photolabile side group. When this group is activated by light, it breaks off leaving the active molecule within the cell.

A

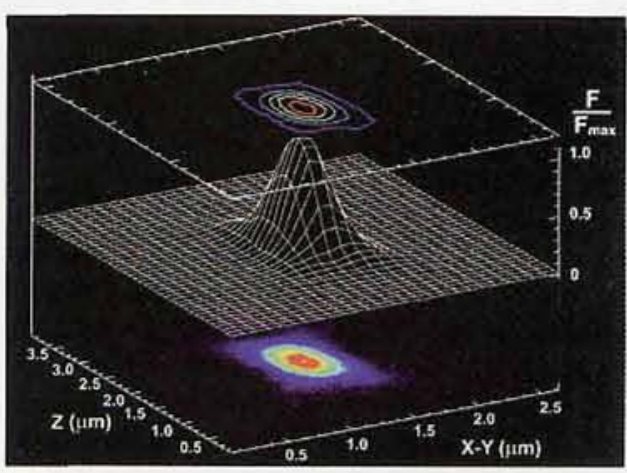

B

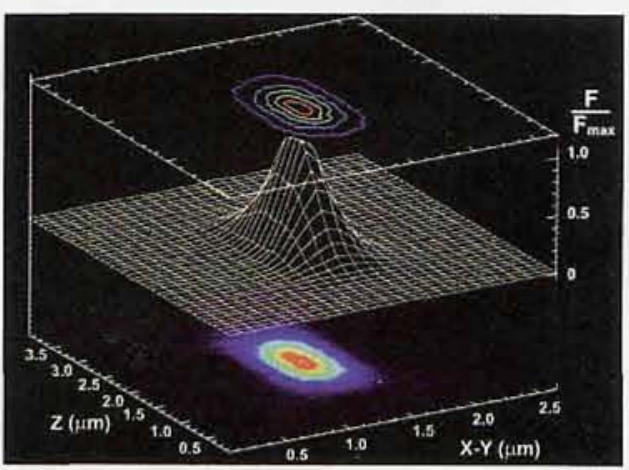

Fig. 4: Imaging performance of our confocal/multi-photon microscope. Panels A and B show the point spread functions measured with sub-resolution fluorescent beads $(0.2$ $\mu \mathrm{m}$ diameter spheres) in conventional confocal and two-photon mode, respectively. The beads were imaged with a Zeiss Plan Neofluar $63 \times 1.25 \mathrm{NA}$ objective. The contour lines show the isophotes from $10 \%$ to $90 \%$ of peak response in steps of $20 \%$. In conventional confocal mode, $x-y$ and axial full width at half maximum resolutions (FWHM) were 0.3 and $0.75 \mu \mathrm{m}$, respectively, with the pinhole set to 1 Airy unit. In two-photon mode (without any pinhole) an $x-y$ FWHM resolution of $0.4 \mu \mathrm{m}$ and an axial resolution of $0.8 \mu \mathrm{m}$ were measured.

\section{Continued on Next Page}




\section{Combined Application of Ultramicrotomy and Scanning Probe Microscopy for Bulk Characteriza- tion Purposes - Continued from previous page.}

Since 2-photon excitation is restricted to the focal plane, it is possible to release the caged compound within a defined volume inside a living cell. The 2-photon microscope is the only instrument that makes such '3-dimensionally resolved' photolysis experiments possible. It is also likely that the development of new fluorescent probes that are more readily excited by 2-photon absorption, will further increase the signal to noise ratio obtained in experiments. Although we are currently using probes that were developed for normal fluorescence excitation and have not yet improved on normal confocal microscopy in terms of resolution and signal-to-noise, such new probes should allow the benefits of 2-photon microscopy to be realised more fully.

Fig. 4 illustrates the imaging performance of our microscope in confocal and 2-photon modes. The resolution of a microscope is defined by the 'point-spread' function that can be measured by imaging a sub-resolution fluorescent bead. This point spread function describes how light from a point source is smeared and distorted by the optical train and can never be smaller than the limit set by the wave properties of light (the 'diffraction limit'). As shown in fig. 4A, the image of a $200 \mathrm{~nm}$ fluorescent sphere in our confocal microscope using a $63 \times 1.25 \mathrm{NA}$ objective is an oval spheroid. This 'lozenge' shape results from the relatively poorer axial (than lateral) performance of the confocal microscope. It should be noted that the axial resolution is mainly provided by the pinhole and, if this element is removed, the image degenerates into a series of rings above and below the plane of the object. This distortion in the image of the object is expected from optical theory and is close to the absolute diffraction limit. The image of the same object using 2-photon excitation is shown in fig. $4 \mathrm{~B}$ and although the pinhole has been removed, the point spread function is comparable to that obtained when using the microscope in confocal mode, despite the fact that the illuminating wavelength was $850 \mathrm{~nm}$ instead of $488 \mathrm{~nm}$.

An unexpected difference between 2-photon and normal fluorescence excitation is that the 2-photon excitation spectra are much wider than their 1-photon counterparts (Xu and Webb, 1996). This means that it is possible to excite several fluorochromes simultaneously with the same (infrared) source tuned to a single wavelength. This completely avoids the problem of longitudinal chromatic aberration which could be a complication when trying to ascertain the relative position of labeled structures within the cell. This point is illustrated in fig. 5 which shows a 3-dimensional representation of the position of two types of cell surface protein in a cultured cell (MDCK epithelial
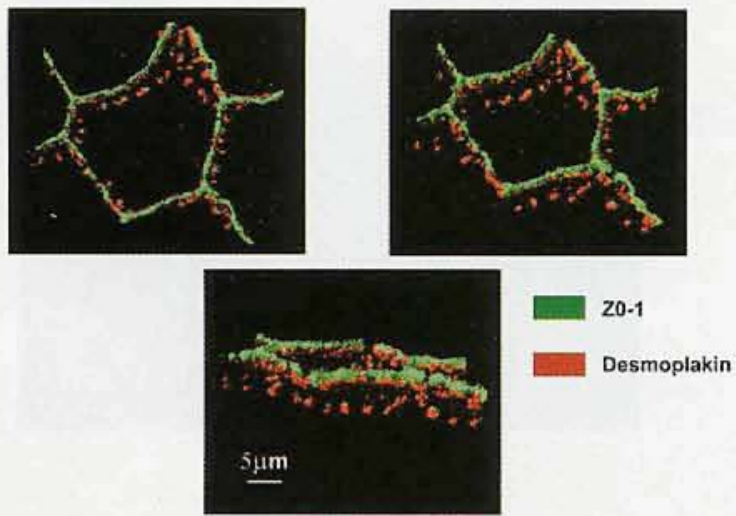

Fig. 5: Volume visualisation of desmoplakin and ZO1 distribution in a monolayer of confluent MDCK cells generated from a closely spaced stack of two-photon images. The images show three different views of the three-dimensional distribution of adhesion proteins that have been visualised by dual labelling with TRITS and FITS linked secondary antibodies. Signals from both fluorochromes were excited by modelocked light from a Ti:S laser at $\sim 840 \mathrm{~nm}$ and simultaneously recorded with two photomultiplier tubes. This ensured absence of any longitudinal chromatic aberration while the three-dimensionally resolved two-photon excitation avoided problems of bleaching. cell line). In this figure, desmoplakin (a protein present in desmosomes) is antibody-labeled with a rhodamine-like compound (TRITC-red). ZO-1 (a protein present tight junctions) is simultaneously labeled with a fluorescein-like compound (FITC -green). Light from the two labels was separated with filters and acquired at the two emission wavelengths simultaneously. The 3-dimensional structure was obtained by taking serial optical sections, a procedure that was helped by the restriction of fluorochrome destruction (bleaching) to the focal plane. In normal fluorescence microscopy, bleaching occurs at the same rate throughout the specimen so it can be difficult to obtain a large enough number of sections for 3-D reconstruction because the later sections may be completely bleached by the prior imaging of the other sections. This would obviously be a serious problem for quantitative measurement of the amount of label present at different heights within the specimen. This problem does not exist in the 2-photon microscope because photodamage is restricted to each focal plane as it is scanned.

To further illustrate the 3-D resolution available from our microscope we have imaged the transverse tubular structures (see Sommer \& Waugh, 1976) within a living cardiac cell. The transverse tubules, which are typically 100-300 $\mathrm{nm}$ in diameter, carry electrical excitation into the interior of the ventricular cell to allow rapid activation throughout the cell (Cheng et al., 1994) and are continuous with the cell exterior. By placing a living cardiac cell in a medium that contained dextran-linked fluorescein, the tubules fill with fluorescein which can be imaged if the massive fluorescence from the fluorescein outside the cell can be rejected or avoided. For such a specimen, the wide field microscope would show almost no detail as the small signal from the fine tubules would be swamped by fluorescence from outside the cell. However, the 2-photon microscope limits excitation to the focal plane and this reduces contamination of the image enormously. As shown in fig. 6 , fine structures within the cell are clearly visible and in fig. 7, a 3-dimensional reconstruction of the tubular system is presented. From such reconstruction, we see that the term 'transverse tubular' system is somewhat a misnomer since the tubules actually form a very complicated reticulum. It should be noted that the tubular system images were also enhanced by computer-based image 'deconvolution' (see above) to correct for the slightly poorer axial response of the microscope. In addition, the 2-photon images have superior contrast when compared to confocal images of the same specimen which may be due to reduced light scattering effects. To our knowledge, these images represent the first visualisation of the entire transverse tubular system in a living cell. Unfortunately, the 3-dimensional complexity of these structures can only be fully appreciated if the data are rotated by a computer in real time, but fig. $7 B$ shows two stereo pairs of images (red-green anaglyph) to allow some

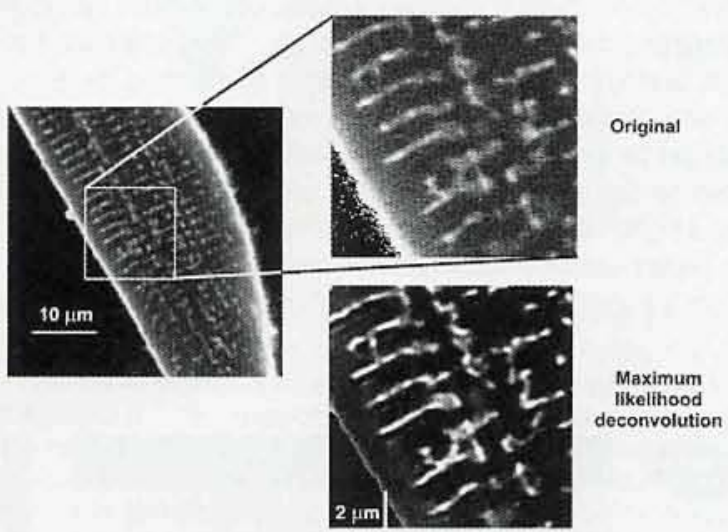

Fig. 6: Imaging of the transverse tubular (t-)system in living rat cardiac myocytes by two-photon microscopy. The left panel shows an "optical slice" of the t-system (visualised by immersing a rat cardiac cell into a bath containing dextran linked fluorescein). The t-system is a network of microscopic tubules (typically $100-300 \mathrm{~nm}$ in diameter). The enlarged view at the upper right shows that the t-system structure can be clearly visualised while fluorescence from the surrounding bathing solution is effectively rejected. To correct for the slightly reduced axial resolution, the three-dimensional image stack formed by sections $0.2 \mu \mathrm{m}$ apart was enhanced by digital deconvolution techniques. The panel on the lower right shows the same detail after application of a maximum likelihood deconvolution procedure which has not only improved the resolution but also enhanced the signal to noise 
appreciation of the 3-dimensional complexity of the tubular system (in a small part of the cell).

In summary, the confocal microscope has allowed us to image sub-cellular signal transduction in living cells with unprecedented temporal and spatial resolution. We have also described the performance of our 2-photon molecular excitation microscope. This instrument indicates that there are some real benefits to this technology which may provide a greater understanding of the 3 dimensional inter-relationship of structures within the cell. In addition, the 2-photon microscope may allow new types of biophysical experiments (on living cells) that require 3-dimensionally resolved fluorescence excitation. With increasing demand, the cost and complexity of mode-locked laser sources should be reduced and the 2-photon microscope should become a powerful new research tool for microscopists and biologists.

Acknowledgements: The authors would like to thank the Wellcome Trust for funding the development of the 2photon microscope and Drs. Robinson and Wang for supplying the specimen shown in fig. 6 . Although this brief article only covers the scientific interests of the authors, several other groups are also actively developing this methodology (e.g., the groups of Watt Webb at Cornell, Winfred Denk at Bell Labs and Dave Piston at Vanderbilt). Cannell, M. B., H. Cheng, and W.J. Lederer. 1994. Spatial non-uniformities in $\left[\mathrm{Ca}^{2+}\right]$ during excitationcontraction coupling in cardiac myocytes. Biophys. J. 67:1942 1956.

Cannell, M. B., H. Cheng, and W.J. Lederer. 1995. The control of calcium release in heart muscle. Science 268:1045-1050

Cheng, H., W. J. Lederer, and M. B. Cannell, 1993. Calcium sparks: Elementary events underlying excitation-contraction coupling in heart muscle. Science 262:740-744.

Cheng, H., W. J. Lederer, and M. B. Cannell. 1994. Propagation

of excitation-contraction coupling into ventricular cells. Pflugers Archiv. 428:415-417.

Denk, W., J. H. Strickler, and W. W. Webb. 1990. Two-photon laser scanning fluorescence microscopy. Science 248:73-76.

López-López, J. R., P. S. Shacklock, C. W. Balke, and W. G. Wier. 1995. Local calcium transients triggered by single L-type calcium channel currents in cardiac cells. Science 268:1042-1045.

Gómez, A.M., Valdivia, H.H., Cheng, H., Lederer, M., Santana, L.F., Cannell, M.B., McCune, S.A., Altshuld, R.A. \& Lederer, W.J. (1997) Defective excitation-contraction coupling in experimental cardiac hypertrophy and heart failure. Science 276: 800-806.

Pawley, J.B. [ed.]. 1995. Handbook of biological confocal microscopy. 2nd ed. Plenum Press, N.Y.

B

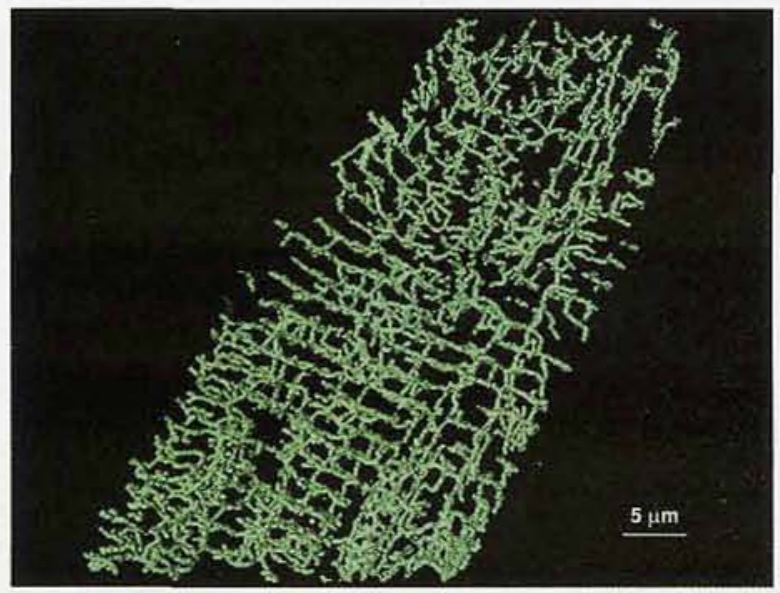

Fig. 7: Volume visualisation of the transverse tubular system in living rat cardiac myocytes. Panel A shows a view of the complicated three-dimensional reticulum forming the transverse system that has been generated from the digitally enhanced image stack (one section of which is shown in fig. 7). Since it is difficult to appreciate the complicated architecture from a two-dimensional view, panel B shows two stereo-pairs (red-green anaglyph) of an enlarged part of the structure.

Soeller, C, and M. B. Cannell. 1996. Construction of a two-photon microscope and optimisation of illumination pulse duration. Pflugers Arch, 432:555-561.

Sommer, J. R. and R. A. Waugh, 1976. The ultrastructure of the mammalian cardiac cell - with special reference on the tubular membrane systems. Am. J. Pathology 82:192-232.

Xu, C., and W. W. Webb. 1996. Measurement of two-photon excitation cross sections of molecular fluorophores with data from 690 to $1050 \mathrm{~nm}$. J. Opt. Soc. Am. B. 13:481-491.

Adapted from the Proceedings of the Royal Microscopical Society, No. 32, pages 3-8, 1997

\section{Job Hunting on the Internet:}

There are many places to hunt for jobs on the internet. Various email servers such as MSA, microprobe, Histonet, etc. carry announcements of open positions. Companies and colleges also carry job openings on their web sites. Below are sites from the www. (While accurate when this was written, some may change as time goes on).

America's Job Bank: Keyword Search:

http://www.aib.dni.us/cgi-bin/websrch.cgi?f_mode=f_kw

Bionet.jobs archive URL:

http://www.bio.net/hypermail/EMPLOYMENT/

Career Related Web Resources:

http://www.geog.psu.edu/ kjordan/jobs/job.htm

CareerPath.com:

http://www.careerpath.com/search.html

The Chronicle of Higher Education - Job Openings:

http://chronicle.merit.edul.ads/.links.html

Employment Opportunities and Resume Postings:

http://doradus,einet.net/GJ/employment.html

J.O.B.S. Database: http://www.careermosaic.com/cm/usenet.html

Job Resources for Microscopists:

http ://www.mwrn.com/feature//joblinks.htm

Jobs in Higher Education - geographical listings:

http://volvo.gslis.utexas.edu/ acadres/jobs/indiv/geog.htm

Monster job search:

http://www.monster.com/

"Nature" (journal) job search:

http://www.america.nature.com/Nature2/serve?SID=21335\&CAT =

Classified\&PG =Jobs/jobsearch.html

"New Scientist" Planet Science: Sciencejobs

http://www.sciencejobs.com/sciencejobs/sciencejobs.html

"Science" (journal) job ads:

http://recruit.sciencemag.org/jobsearch.shtml

SuperSite tech jobs:

http://SuperSite.Net/TechJobs/

U.S. Federal Government - Current Job Openings:

http://www.usajobs.opm.gov/a.htm

Usenet newsgroup:

bionet.jobs.offered 